\title{
Peripheral Biomarkers for Alzheimer's Disease: Update and Progress
}

Marwan N. Sabbagh · Kaj Blennow

Received: November 11, 2019 /

(C) The Author(s) 2019

In 1984, the NINDS-ADRDA published criteria stating that definite Alzheimer's disease (AD) could only be confirmed by biopsy or autopsy [1]. This publication led to the widely held perception that $\mathrm{AD}$ diagnosis was a diagnosis of exclusion only. Since then, the field has evolved rapidly with the advent of cerebrospinal fluid (CSF) testing for AD with great sensitivity and specificity [2], and the development of amyloid positron emission tomography (PET), tau PET, and fluorodeoxyglucose PET. Amyloid PET has emerged as an added value test that could alter clinical management $[3,4]$. Magnetic resonance imaging (MRI) is also evolving as a tool to measure regional volumes, particularly within the hippocampus, as a proxy measure of neurodegeneration. Many of these tests have excellent specificity and sensitivity but are limited by access/availability of such technologies

Enhanced Digital Features To view enhanced digital features for this article go to https://doi.org/10.6084/ m9.figshare.10283069.

M. N. Sabbagh $(\bowtie)$

Cleveland Clinic Lou Ruvo Center for Brain Health, Las Vegas, NV, USA

e-mail: sabbagm@ccf.org

K. Blennow

Clinical Neurochemistry Laboratory, Department of Psychiatry and Neurochemistry, Institute of

Neuroscience and Physiology, University of

Gothenburg, Gothenburg, Sweden as PET, the expense of tests, and the invasive quality of lumbar puncture.

Ideally, a peripheral biomarker could be used as a screening tool, with the driver being the negative predictive value, similar to amyloid PET imaging. This type of biomarker would be advantageous for its minimal invasiveness and potentially lower cost. Specifically, a peripheral diagnostic tool with a normal value would reflect the absence of the target pathology, while an abnormal value would prompt further investigation using PET and MR imaging techniques, or CSF tests. It could be valuable as a screening tool in therapeutic and prevention trials and considerably reduce the costs of ruling out potential participants who do not have the target pathology. Screening tests also would be valuable in the primary care setting when disease-modifying therapies become available.

Exploration into the development of peripheral biomarkers has accelerated rapidly in recent years. This acceleration is partly due to technological developments, with ultra-sensitive methods lowering the lower limit of quantification from the nanogram per liter range to the picogram per liter range. Thus, proteins previously thought to be undetectable in peripheral samples are now measurable. In this special issue, we include seven innovative and potentially scalable peripheral diagnostic tests currently in development. 
In the first contribution, Lue et al. [5] provide a review and summary of the immunomagnetic reduction base SQUID (superconducting quantum interference device)) technology. Immunomagnetic reduction (IMR) is emerging as one of these ultrasensitive technologies. The IMR biomarker detection system uses antibody-conjugated magnetic nanoparticles as bioprobes and a high-temperature, AC (alternating current) magneto-susceptometer SQUID to detect the amounts of antigens bound to the bioprobes $[6,7]$. Recent studies indicate the detection of significant increases of amyloid beta $42\left(\mathrm{~A} \beta_{42}\right)$ and total tau (T-tau) levels in clinically-diagnosed early AD subjects when compared with cognitively normal control subjects.

In the second contribution, Ashton et al. [8] review the use of saliva as a peripheral biospecimen. Our group has shown that $A \beta$ was detectable and elevated in the saliva of $A D$ subjects [9]. Ashton et al. point out that saliva, however, is also a rich source of potential biomarkers for disease detection and offers several practical advantages over biofluids that are currently being examined for neurodegenerative disorders. However, the collection of saliva in the elderly is challenging, including changes in the production and composition of saliva as age advances. The authors point out that $\mathrm{A} \beta, \alpha$ synuclein, and tau species are among the many proteins detectable in saliva.

In the third contribution, Smailovic and Jelic [10] review the progress of applying electroencephalography (EEG) as an AD biomarker. EEG has been around for decades and was explored in the past for signatures of neurodegenerative diseases. Previous research suggested that EEG lacks sensitivity, although it was routinely used as part of the diagnostic evaluation. However, new-generation technology has propelled reconsideration. Resting-state EEG is a widely available and noninvasive diagnostic method that provides direct insight into brain synaptic activity in real time. Quantitative EEG (qEEG) analysis additionally provides information on physiologically meaningful frequency components, dynamic alterations, and topography of EEG signal generators, i.e., neuronal signaling. Numerous studies have shown that qEEG measures can detect disruptions in activity, topographical distribution, and synchronization of neuronal (synaptic) activity, such as generalized EEG slowing, reduced global synchronization, and anteriorization of neuronal generators of fast-frequency resting state EEG activity in patients along the AD continuum. Moreover, qEEG measures appear to correlate well with surrogate markers of $\mathrm{AD}$ neuropathology and discriminate between different types of dementia. Because EEG is fairly ubiquitous, it could be easily deployed as a tool to detect neurodegenerative disease.

In the fourth contribution, $\mathrm{Li}$ and Mielke [11] review the detection of plasma protein using the single molecular array (SiMoA) platform. SiMoA is an ultrasensitive technology that can detect proteins in blood at sub-femtomolar concentrations (i.e., $10^{-16} \mathrm{M}$ ). Li and Mielke focus on the utility of SiMoA assays for the measurement of plasma or serum $\mathrm{A} \beta_{42}$, phosphorylated tau (p-tau), T-tau, and neurofilament light chain (NfL). This is one of the leading platforms being developed for use in screening asmA proteins.

In the fifth contribution, Oeckl and Otto [12] review mass spectometry (MS) as a third detection method for plasma proteins. MS has for many years been used as a sensitive detection method and is an established tool for the measurement of various analytes in biological fluids, such as blood. Its greatest strength is its high selectivity. Importantly, results from recent MS studies correlate highly with those from studies using amyloid PET, suggesting that MS detects target pathology [13]. Efforts to scale and commercialize this technology are currently taking place.

In the sixth contribution, Vanderstichele et al. [14] provide a cautionary note about the deployment of blood-based biomarker assays in clinical applications. These observations include technological considerations for assay development and critical raw materials for $A \beta$ and tau quantification in blood. They propose improved workflows for design, development, and validation of (immuno)assays for blood proteins.

In the final contribution, Rissman et al. [15] provide insights into recent developments in 
the area of retinal scanning, which may serve as a non-invasive and simple method to identify AD. Retinal scanning can identify vascular abnormalities as well as changes in the early AD disease process indicative of inflammation. Some studies also indicate its potential to detect $\mathrm{A} \beta$ inclusions. The authors also call for caution, given the small sample sizes and variability in measures in published papers, as well as possible confounders, such as existing retinopathy and aging effects in the retina, which may limit its performance for AD identification, as well as the need to compare it with established biomarkers.

In summary, the papers presented in this special issue give the reader a comprehensive and up-to-date overview of the rapidly expanding field of peripheral biomarkers for AD. These biomarkers offer hope for the future availability of easily accessible and cost-effective screening tools that can be used in the clinical management and diagnostic evaluation of patients with suspected early AD.

\section{ACKNOWLEDGEMENTS}

This study was supported by National Institute on Aging (5P20GM109025 and Keep Memory Alive Foundation).

Funding. No funding or sponsorship was received for this study or publication of this article.

Authorship. All named authors meet the International Committee of Medical Journal Editors (ICMJE) criteria for authorship for this article, take responsibility for the integrity of the work as a whole, and have given their approval for this version to be published.

Disclosures. MN Sabbagh served as an investigator in the biomarker development plan. He has no proprietary interest in the investigational products or the company. Kaj Blennow is supported by the Torsten Söderberg Foundation, Stockholm, Sweden and has served as a consultant or on advisory boards for Alector, Alzheon, CogRx, Biogen, Lilly, Novartis, and Roche Diagnostics; he is also a co-founder of Brain Biomarker Solutions in Gothenburg AB.

Compliance with Ethics Guidelines. This article is based on previously conducted studies and does not contain any studies with human participants or animals performed by any of the authors.

Open Access. This article is distributed under the terms of the Creative Commons Attribution-NonCommercial 4.0 International License (http://creativecommons.org/licenses/ by-nc/4.0/), which permits any noncommercial use, distribution, and reproduction in any medium, provided you give appropriate credit to the original author(s) and the source, provide a link to the Creative Commons license, and indicate if changes were made.

\section{REFERENCES}

1. McKhann G, Drachman D, Folstein M, Katzman R, Price D, Stadlan EM. Clinical diagnosis of Alzheimer's disease: report of the NINCDS-ADRDA Work Group under the auspices of Department of Health and Human Services Task Force on Alzheimer's Disease. Neurology. 1984;34(7):939-44. https://doi. org/10.1159/000478007. Epub 2017 Aug 5.

2. Olsson B, Lautner R, Andreasson U, et al. CSF and blood biomarkers for the diagnosis of Alzheimer's disease: a systematic review and meta-analysis. Lancet Neurol. 2016;15(7):673-84. https://doi.org/ 10.1016/S1474-4422(16)00070-3.

3. Pontecorvo MJ, Siderowf A, Dubois B, et al. Effectiveness of florbetapir pet imaging in changing patient management. Dement Geriatr Cogn Disord. 2017;44(3-4):129-43. https://doi.org/10.1159/ 000478007. Epub 2017 Aug 5.

4. Rabinovici GD, Gatsonis C, Apgar C, et al. Association of amyloid positron emission tomography with subsequent change in clinical management among medicare beneficiaries with mild cognitive impairment or dementia. JAMA. 2019;321(13): 1286-94. https://doi.org/10.1001/jama.2019.2000.

5. Lue L, Kuo Y, Sabbagh MN. Advances in plasma AD core biomarker development: current findings from immuno-magnetic reduction-based SQUID technology. Neurol Ther. 2019. https://doi.org/10.1007/ s40120-019-00167-2. 
6. Chiu MJ, Yang SY, Chen TF, et al. New assay for old markers-plasma beta amyloid of mild cognitive impairment and Alzheimer's disease. Curr Alzheimer Res. 2012;9:1142-8.

7. Huang KW, Yang SY, Hong YW, et al. Feasibility studies for assaying alpha-fetoprotein using antibody-activated magnetic nanoparticles. Int $\mathrm{J}$ Nanomed. 2012;7:1991-6. https://doi.org/10.2147/ IJN.S28245. Epub 2012 Apr 17.

8. Ashton N, Ide M, Zetterberg H, Blennow K. Salivary biomarkers for Alzheimer's disease and related disorders. Neurol Ther. 2019. https://doi.org/10.1007/ s40120-019-00168-1.

9. Sabbagh MN, Shi J, Lee M, et al. Salivary beta amyloid protein levels are detectable and differentiate patients with Alzheimer's disease dementia from normal controls: preliminary findings. BMC Neurol. 2018;18(1):155. https://doi.org/10.1186/ s12883-018-1160-y.

10. Smailovic U, Jelic V. Neurophysiological markers of Alzheimer's disease: quantitative EEG approach. Neurol Ther. 2019. https://doi.org/10.1007/s40120019-00169-0.
11. Li D, Mielke M. An update on blood-based markers of Alzheimer's disease using the SiMoA platform. Neurol Ther. 2019. https://doi.org/10.1007/s40120019-00164-5.

12. Oeckl P, Otto M. A review on MS-based blood biomarkers for Alzheimer's disease. Neurol Ther. 2019. https://doi.org/10.1007/s40120-019-00165-4.

13. Nakamura A, Kaneko N, Villemagne VL, et al. High performance plasma amyloid- $\beta$ biomarkers for Alzheimer's disease. Nature. 2018;554(7691):249-54. https://doi.org/10.1038/nature25456. Epub 2018 Jan 31.

14. Vanderstichele et al. Critical steps to be taken before quantification $ß$-amyloid isoforms in blood can be implemented in a clinical environment. Neurol Ther. 2019. https://doi.org/10.1007/s40120019-00166-3.

15. Rissman R. Reflections on the utility of the retina as a biomarker for Alzheimer's disease. Neurol Ther. 2019. https://doi.org/10.1007/s40120-019-00173-4. 